
\title{
$\begin{array}{ll}\text { Research Square } & \begin{array}{l}\text { Preprints are preliminary reports that have not undergone peer review. } \\ \text { They should not be considered conclusive, used to inform clinical practice, } \\ \text { or referenced by the media as validated information. }\end{array}\end{array}$
}

\section{Preliminary study of the use of a CBT screw to treat lumbar adjacent segment degeneration}

\section{Yonghui Zhao ( $\square$ axliu1027@163.com )}

People's Liberation Army Joint Logistic Support Force 920th Hospital https://orcid.org/0000-0002-4802-5456

Jinlong Liang

People's Liberation Army Joint Logistic Support Force 920th Hospital

Haotian Luo

People's Liberation Army Joint Logistic Support Force 920th Hospital

\section{Yulong Ma}

People's Liberation Army Joint Logistic Support Force 920th Hospital

\section{Taibang Chen}

People's Liberation Army Joint Logistic Support Force 920th Hospital

\section{Zhaowei Teng}

Kunming Medical University

\section{Zhi Peng}

Kunming Medical University

\section{Yongqing Xu}

People's Liberation Army Joint Logistic Support Force 920th Hospital

\section{Sheng Lu}

First People's Hospital of Yunnan

\section{Research article}

Keywords: Lumbar vertebrae, CBT Screw, Pedicle Screw, Adjacent Segmental Disease

Posted Date: November 26th, 2019

DOI: https://doi.org/10.21203/rs.2.17729/v1

License: (c) (i) This work is licensed under a Creative Commons Attribution 4.0 International License. Read Full License 


\section{Abstract}

Objective: To investigate the feasibility of using the cortical bone trajectory (CBT) screw in revision surgery for lumbar adjacent segment degeneration (LASD) and to provide a reference for clinical practice.

Methods:The computed tomography (CT) scans of the lumbar spine of 40 patients in our hospital were used. Threedimensional (3D) reconstruction was performed using Mimics 19.0 software, screws with appropriate sizes were selected for the L1 to L5 vertebral segments, and the traditional pedicle (TP) screws were placed using the standard method. After the completion of screw placement, the simulated placement of CBT screws with appropriate sizes was performed separately. During screw placement, the site and the direction were adjusted accordingly to complete the screw placement as much as possible. Under the premise of safe and feasible placement, penetration of the screw through the CBT and overlap between the TP and CBT screws should be avoided. If these requirements are met, screw placement is considered successful. Otherwise, the screw placement is considered failed. After all simulated screw placements were complete, the success rate of the screw placement in each segment of the lumbar vertebra was calculated from the statistical results. Finally, the pedicle trajectory reference width (TRW) was used for grouping to investigate the correlation between the TRW and sucess rate of screw placement.

Results: Four hundred simulated screw placements (80 in each pedicle) were performed in the L1-L5 pedicles. The overall success rate of CBT screw placement in the lumbar spine was $57.00 \%$. The success rates in the L1 to L5 segments were $47.50 \%, 62.50 \%, 57.50 \%, 70.00 \%$, and $47.50 \%$, respectively. The success rate in L4 was greater than in L2 and L3, while the lowest success rates were observed in L1 and L5. The groups were established based on the TRW. The success rate of CBT screw placement increased as the TRW increased.

Conclusions: CBT screws are not suitable treatments for all cases of LASD. The success rate of screw placement varies in different lumbar segments. A preoperative measurement of TRW has important reference value for evaluating the feasibility of CBT screw placement in revision surgery for LASD.

\section{Background}

With the aging of the world population and the development of the pedicle screw technique, the number of patients undergoing lumbar fusion with internal fixation has increased rapidly each year. Between 1994 and 2006, the National Survey of Ambulatory Surgery (NSAS) revealed 5.4- and 2.6-fold increases in the number of surgeries for patients with intervertebral disc disease and spinal stenosis, respectively, and the number of patients requiring intervertebral fusion surgery increased by $340 \%$ [1]. Deyo [2] analyzed the National Health Data from the Online Health Care Utilization Project and found that the number of lumbar fusion surgeries conducted between 1996 and 2011 increased by $600 \%$. However, spinal fusion changes the biomechanical environment of the spine and stresses the intervertebral disc and facet joints of adjacent segments, thereby accelerating the degeneration of adjacent segments and resulting in secondary lumbar disc herniation, lumbar spinal stenosis and degenerative lumbar spondylolisthesis. This phenomenon is called lumbar adjacent segment degeneration (LASD), and patients with severe symptoms must be treated with revision surgery $[3,4]$. The revision of LASD often requires the removal of the original internal fixation devices, resulting in a prolonged operative time, intraoperative blood loss, many postoperative complications, and increased difficulty and risks of surgery, posing substantial challenges to many surgeons [5-6].

The advent of CBT screw provides a new option for the revision of LASD. The CBT screws are usually placed at farther medial and inferior sites of the pedicle. The direction of the screw placement is from bottom up in the sagittal 
plane through the pedicle, and the screw is placed from inside to outside in the cross section. This placement is different from the trajectory of traditional pedicle (TP) screw placement. Therefore, bridging fixation is possible to achieve with CBT screws without removing the pedicle screw internal fixation device, and thereby the revision of LASD is achieved. The CBT screw has the advantages of inducing less trauma and less bleeding, a short operative time, and fewer postoperative complications. However, this procedure is difficult to perform, and few reports are available in the literature. Several questions remain. Can CBT screws be used for revision surgery to treat LASD? What is the success rate of the revision surgery using CBT screws? What are the main factors affecting the success rate of the revision surgery? Therefore, in this study, we employed digital technology to explore the feasibility of this method by performing simulated operations to provide a reference for clinical applications.

\section{Materials And Methods}

\subsection{General data}

All data used in this study were approved by the Ethics Committee of the 920 Hospital of the Joint Logistic Support Force, People's Liberation Army of China. The computed tomography (CT) scan data were obtained from 40 hospitalized patients in our hospital, including 18 males and 22 females with an average age of $57.90 \pm 17.40$ years (range: $19-83$ years). The inclusion criteria were $\square$ patients aged greater than 18 years and $\square$ patients with complete lumbar CT scan data. The exclusion criteria were $\square$ patients with a lumbar deformity or vertebral dysplasia, $\square$ patients with damaged vertebral structures due to fractures, infections or tumors, and $\square$ patients with a history of lumbar surgery.

\subsection{Study methods}

\subsubsection{Selection of the screw size}

The lumbar CT scan data from 40 patients were imported into Mimics 19.0 software for the threedimensional (3D) reconstruction in the Digital Imaging and Communications in Medicine (DICOM) format. Since the height of the L1-L5 pedicles is greater than the width, the pedicle width is the main factor limiting the diameter of the screw. Therefore, we first measured the pedicle trajectory reference width (TRW), pedicle spongy width (SBW) and pedicle width (PW), as shown in Figure 1. The TRW was the main parameter used to select the diameter of CBT screws. When the TRW is between $4.0 \mathrm{~mm}$ and $4.5 \mathrm{~mm}$, a screw with a diameter of $4.0 \mathrm{~mm}$ is recommended. When the TRW is between $4.5 \mathrm{~mm}$ and $5.0 \mathrm{~mm}$, a screw with a diameter of $4.5 \mathrm{~mm}$ is recommended. When the TRW is between $5.0 \mathrm{~mm}$ and $5.5 \mathrm{~mm}$, a screw with a diameter of $5.0 \mathrm{~mm}$ is recommended. When the TRW is greater than $5.5 \mathrm{~mm}$, a screw with a diameter of $5.5 \mathrm{~mm}$ is recommended. The SBW and PW were the reference parameters used to select the diameter of TP screws. When considering the placement of both CBT screws and TP screws in the same pedicle, we selected screws with appropriate diameters based on the relevant parameters measured (Table 1).

[Figure 1] 
Table 1 Related parameters and diameters of the screws in simulated placements

\begin{tabular}{|c|c|c|c|c|c|}
\hline \multirow[t]{2}{*}{ Lumbar segment } & \multicolumn{3}{|c|}{ Related parameters of pedicle trajectory (mm) } & \multicolumn{2}{|c|}{ Screw diameter $(\mathrm{mm})$} \\
\hline & TRW & PW & TRW & $\mathrm{TP}$ & CBT \\
\hline L1 & $4.6 \pm 1.1$ & $7.7 \pm 1.3$ & $4.1 \pm 1.1$ & 5.5 & 4.0 \\
\hline L2 & $5.8 \pm 1.2$ & $8.7 \pm 1.5$ & $4.7 \pm 0.9$ & 6.0 & 4.0 \\
\hline L3 & $7.2 \pm 1.1$ & $10.1 \pm 1.2$ & $5.8 \pm 0.9$ & 6.5 & 4.5 \\
\hline L4 & $9.2 \pm 1.4$ & $12.4 \pm 1.5$ & $7.1 \pm 1.2$ & 6.5 & 5.0 \\
\hline L5 & $15.9 \pm 1.7$ & $6.6 \pm 1.0$ & $6.6 \pm 1.0$ & 6.5 & 5.0 \\
\hline
\end{tabular}

Notes: Cortical bone trajectory (CBT) and traditional pedicle (TP).

First, TP screws were placed in the L1 to L5 pedicles. The criteria for screw placement are described below. $\square$ The vertex of the " $\lambda$ "-shaped ridge was selected as the entry point for screw placement. $\square$ Regarding the direction of screw placement, the screw trajectory in the in the sagittal plane was close to the upper edge of the pedicle and parallel to the endplate of the vertebral body. The screw was placed along the pedicle axis in the cross section, and the depth was $1 / 3$ of the vertebral body. $\square$ The diameter of the screw was $5.0 \mathrm{~mm}$ in L1, $6.0 \mathrm{~mm}$ in L2, $6.5 \mathrm{~mm}$ in L3, $6.5 \mathrm{~mm}$ in L4, and $6.5 \mathrm{~mm}$ in L5 (Figure 2). After TP screw placement, the TP pedicle screw parameters were maintained unchanged, and CBT screw placement was simulated. The diameters of the CBT screws were $4.0 \mathrm{~mm}$ in $\mathrm{L} 1,4.0 \mathrm{~mm}$ in $\mathrm{L} 2,4.5 \mathrm{~mm}$ in $\mathrm{L} 3,5.0 \mathrm{~mm}$ in $\mathrm{L} 4$, and $5.0 \mathrm{~mm}$ in $\mathrm{L} 5$. The CBT screw placement direction was from bottom to top and from inside to outside and was adjusted appropriately according to the position of pedicle screw to maximize the completion of CBT screw placement (Figure 3). Under the premise of safe and feasible placement, penetration of the screw through the CBT and overlap between the TP and CBT screws should be avoided. If these requirements were met, the screw placement was considered successful. During the simulated screw placement, the screw penetration through the CBT or overlap between the TP and CBT screws was inevitable, although the CBT screw was adjusted (Figure 4). After the completion of simulated screw placements, the numbers of successful and failed screw placements were counted. The overall success rate of the total simulated screw placements in the lumbar vertebrae and the success rate of each segment were calculated. Finally, the pedicle TRW was used for grouping to investigate the correlation between the TRW and success rate of screw placement in each group.

[Figure 2, 3, 4]

\subsection{Statistical analysis}

SPSS 22 statistical software (SPSS, USA) was used for statistical analyses. The KolmogorovSmirnov test was used to test the normal distribution of the quantitative data. Quantitative data with normal distribution are presented as $\pm s$ and were compared using a random analysis of variance. $\mathrm{P}<0.05$ was considered statistically significant. 


\section{Results}

In this study, 400 simulated screw placements ( 80 for each pedicle) were performed in lumbar pedicles. The results are described below. In L1, 38 successful screw placements and 42 failed screw placements were recorded, with a success rate of $47.50 \%$. Fifty successful screw placements and 30 failed screw placements were recorded in L2, with a success rate of $62.50 \%$. In L3, 46 successful screw placements and 34 failed screw placements occurred, with a success rate of $57.5 \%$. Fifty-six successful screw placements and 24 failed screw placements were recorded in L4, with a success rate of $70 \%$. In L5, 38 successful screw placements and 42 failed screw placements were recorded, with a success rate of $47.5 \%$.

The groups were established based on the TRW. The success rate of CBT screw placement increased as the TRW increased. When the TRW was increased to a certain level, the success rate of the screw placement increased significantly. When the TRW was greater than $5.0 \mathrm{~mm}$, the success rate of screw placement was $\geq 85.71 \%$ in $\mathrm{L} 1$ and $\geq 83.33 \%$ in L2. When the TRW was greater than 6.0 $\mathrm{mm}$, the success rate of screw placement was $\geq 84.62 \%$ in $\mathrm{L} 3$ and $\geq 81.82 \%$ in L4. The success rate of screw placement was $\geq 87.50 \%$ in L5 when the TRW was greater than $7.5 \mathrm{~mm}$. Therefore, the feasibility of revision surgery using CBT screws was evaluated based on the TRW.

Table 2 Summary of screw placement in the lumbar vertebrae after grouping based on the TRW

\begin{tabular}{|c|c|c|c|c|c|c|c|c|c|c|c|c|c|c|c|c|c|c|c|c|}
\hline \multirow{2}{*}{$\begin{array}{l}\text { TRW } \\
\text { Group }\end{array}$} & \multicolumn{4}{|c|}{ L1 } & \multicolumn{4}{|c|}{ L2 } & \multicolumn{4}{|c|}{ L3 } & \multicolumn{4}{|c|}{ L4 } & \multicolumn{4}{|c|}{ L5 } \\
\hline & $\mathrm{S}$ & $\mathrm{F}$ & $\mathrm{n}$ & $\mathrm{S} \%$ & $\mathrm{~S}$ & $\mathrm{~F}$ & $\mathrm{n}$ & $\mathrm{S} \%$ & $\mathrm{~S}$ & $\mathrm{~F}$ & $\mathrm{n}$ & $\mathrm{S} \%$ & $\mathrm{~S}$ & $\mathrm{~F}$ & $\mathrm{n}$ & $\mathrm{S} \%$ & $\mathrm{~S}$ & $\mathrm{~F}$ & $\mathrm{n}$ & $\mathrm{S} \%$ \\
\hline$\square 4.0$ & 0 & 23 & 23 & $0.00 \%$ & 0 & 11 & 11 & $0.00 \%$ & 0 & 4 & 4 & $0.00 \%$ & 0 & 1 & 1 & $0.00 \%$ & & & 0 & \\
\hline$[4.0,4.5 \square$ & 4 & 9 & 13 & $30.77 \%$ & 2 & 10 & 12 & $20.00 \%$ & 0 & 4 & 4 & $0.00 \%$ & 0 & 1 & 1 & $0.00 \%$ & & & 0 & \\
\hline$[4.5,5.0 \square$ & 12 & 8 & 20 & $60.00 \%$ & 7 & 5 & 12 & $58.33 \%$ & 1 & 5 & 6 & $16.67 \%$ & & & 0 & & 0 & 2 & 2 & $0.00 \%$ \\
\hline$[5.0,5.5 \square$ & 12 & 2 & 14 & $85.71 \%$ & 15 & 3 & 18 & $83.33 \%$ & 3 & 8 & 11 & $27.27 \%$ & 0 & 1 & 1 & $0.00 \%$ & 0 & 3 & 3 & $0.00 \%$ \\
\hline$[5.5,6.0 \square$ & 10 & 0 & 10 & $100 \%$ & 17 & 1 & 18 & $94.44 \%$ & 16 & 11 & 27 & $59.26 \%$ & 2 & 9 & 11 & $18.18 \%$ & 2 & 15 & 17 & $11.76 \%$ \\
\hline$[6.0,6.5 \square$ & & & 0 & & 9 & 0 & 9 & $100 \%$ & 11 & 2 & 13 & $84.62 \%$ & 7 & 3 & 10 & $70.00 \%$ & 8 & 13 & 21 & $38.10 \%$ \\
\hline$[6.5,7.0 \square$ & & & 0 & & & & 0 & & 5 & 0 & 5 & $100 \%$ & 5 & 4 & 9 & $55.56 \%$ & 7 & 4 & 11 & $63.64 \%$ \\
\hline$[7.0,7.5 \square$ & & & 0 & & & & 0 & & 5 & 0 & 5 & $100 \%$ & 18 & 4 & 22 & $81.82 \%$ & 8 & 4 & 12 & $66.67 \%$ \\
\hline$[7.5,8.0]$ & & & 0 & & & & 0 & & 5 & 0 & 5 & $100 \%$ & 8 & 1 & 9 & $88.89 \%$ & 7 & 1 & 8 & $87.50 \%$ \\
\hline$\geq 8.0$ & & & 0 & & & & 0 & & & & 0 & & 16 & 0 & 16 & $100 \%$ & 6 & 0 & 6 & $100 \%$ \\
\hline & 38 & 42 & 80 & $47.50 \%$ & 50 & 30 & 80 & $62.50 \%$ & 46 & 34 & 80 & $57.50 \%$ & 56 & 24 & 80 & $70.00 \%$ & 38 & 42 & 80 & $47.50 \%$ \\
\hline
\end{tabular}

Notes: S: successful placement, F: failed placement, n: sample size, S\%: success rate of placement, TRW: trajectory reference width

\section{Discussion}

Pedicle screw internal fixation is currently the most commonly used and most reliable fixation method in spinal surgery. However, interbody fusion accelerates the degeneration of adjacent segments [7], leading to the occurrence of LASD. The incidence of LASD varies between studies. According to imaging evidence, the prevalence of LASD exceeds $40 \%$, and the rate of surgical interventions ranges from $5.2 \%$ to $18.5 \%$ [7-8]. LASD surgery often requires the removal of the original internal fixation devices, which not only increases the exposure range of the operative field, prolongs the operative time and increases the blood loss and surgical trauma but also increases the risk of postoperative infections. The advent of the CBT screw provides a new option for the revision of LASD. The revision 
can be completed by bridge fixation of the adjacent vertebral body using CBT screws to avoid the limitations of the conventional revision surgery. In 2014, Rodriguez et al. [9] used an 0-arm navigation system to complete the revision of LASD in 5 patients by placing CBT screws in the pedicle of the upper fused vertebra without removing the original internal fixation device. Wang et al. [10] also reported satisfactory results for 12 patients with lumbar spondylosis who underwent revision surgery with CBT screws without removing the internal fixation device. Chen et al. [11] performed revision surgery on 6 patients with LASD using the C-arm machine alone, and satisfactory results were also achieved. Based on these studies, CBT screws can be used for revision surgery in patients with LASD; however, related reports are rare in the literature. Due to the difference in the pedicle anatomy of different segments of the lumbar spine, the same lumbar segment displays some differences between individuals. Can CBT screws be used for the revision of LASD in patients? What is the success rate of lumbar revision surgery? What are the factors that affect the success rate of screw placement? The use of the CBT screw in bridge fixation to complete the revision surgery has been a challenge in an increasing number of patients with LASD.

Mullin et al. [12] selected lumbar CT scan data from 47 patients and divided them into two groups according to the history of lumbar surgery. In one group, CBT screws were placed based on the locations of the original pedicle screws. In the other group, two types of screws were placed concurrently. The authors reported the feasibility of inserting 2 screws at the same time. The results obtained from the study have very limited value because of the nonuniform distribution of the surgical fixated segments in the group with a surgical history, namely, only 3 patients underwent original fixation in L1. In the present study, we used digital technology to simulate the CBT screw placement based on the TP screws and verified the feasibility of the CBT screw placement. Using this approach, we successfully solved the problem of the "non-uniform distribution of surgical fixated segments" in the study by Jeffrey et al.

In the present study, lumbar CT scan data from 40 patients were used for simulated screw placement after 3D reconstruction. According to the TRW, the diameter of the selected CBT screw was 4.0-5.0 mm, and the diameter of the pedicle screw was 5.5-6.5 $\mathrm{mm}$. These screw sizes are not the same as those selected in the previous studies. Ueno et al. [13] used CBT screws with a diameter of $4.5 \mathrm{~mm}$ (L1-L5: $4.5 \mathrm{~mm}$ ), and TP screws with a diameter of 5.5$6.5 \mathrm{~mm}$ (L1-L2: $5.5 \mathrm{~mm}$ and L3-L5: $6.5 \mathrm{~mm}$ ). Rodriguez et al. [9] selected CBT screws with a diameter of $5.5 \mathrm{~mm}$ and TP screws with a diameter of 7.0 to $7.5 \mathrm{~mm}(L 1, L 2$ and $L 4: 7.0 \mathrm{~mm}$ and $L 3$ and $L 5: 7.5 \mathrm{~mm}$ ) for revision surgery. Different screw sizes might exert different effects on the success rate and biomechanical strength. Screws that are too large might also increase the risk of fracture of the pedicle isthmus. Further studies are needed to validate these hypotheses. Based on conventional criteria, we simulated the TP screw placement and then the CBT screw placement. We achieved reproducible results using this strategy. The penetration of the CBT screw was directly viewed. The overlap of the 2 screws is viewed by concealing the vertebral body or making the vertebral body transparent. Therefore, the successful placement of the screws is easy to determine. In addition, the problem of an insufficient sample size in the study was also avoided. However, the design has certain limitations. The pedicle screws are placed according to the conventional standard, but the angle of the pedicle screw placed in the actual clinical setting is uneven due to the difference between the screw placement methods and the surgeon's preferences. Therefore, the actual accuracy of screw placement is different. Similarly, the success rate of screw placement varies in different lumbar segments. PH and SBW are important parameters for selecting TP screws, while the TRW is an important parameter for selecting the diameter of CBT screws. Therefore, we completed the simulated screw placement and then used TRW for grouping to investigate screw placement in different segments. Notably, the success rate of CBT screw placement increased as the TRW increased. Therefore, a preoperative measurement of TRW will provide general information about whether CBT screws can be used for revision surgery. In subsequent studies, we will consider a classification based on the positions of pedicle screws in the pedicle to further improve

Page 6/11 
the success rate of screw placement. In addition, we also plan to perform 3D reconstruction based on the patient's initial lumbar CT scan data and simulate the screw placement according to current criteria of the position and the size of the vertebral screw. Then, based on the position of the pedicle screw, we will simulate the CBT screw placement to provide a reference for the operation. A guide template based on the CBT can be designed as an intraoperative assistant to maximize the screw placement.

As shown in the present study, the CBT screw is not suitable for all patients with LASD who require revision surgery. The success rate of screw placement varies in different lumbar segments. A preoperative measurement of TRW can predict success rates in different lumbar segments and provides an important reference value for evaluating the feasibility of using CBT screws in revision surgery for LASD.

\section{References}

1. Best M J, Buller L T, Eismont F J. National Trends in Ambulatory Surgery for Intervertebral Disc Disorders and Spinal Stenosis: A 12-Year Analysis of the National Surveys of Ambulatory Surgery[J]. Spine, 2015, 40(21).

2. Deyo R A. Fusion surgery for lumbar degenerative disc disease: still more questions than answers. [J]. Spine Journal Official Journal of the North American Spine Society, 2015, 15(2):272-274.

3. Madan S S, Boeree N R. Comparison of instrumented anterior interbody fusion with instrumented circumferential lumbar fusion[J]. European Spine Journal, 2003, 12(6):567-575.

4. Yu S W, Yen C Y, Wu C H, et al. Radiographic and clinical results of posterior dynamic stabilization for the treatment of multisegment degenerative disc disease with a minimum follow-up of 3 years[J]. Archives of Orthopaedic and Trauma Surgery, 2012, 132(5):583-589.

5. Owens R K, Djurasovic M, Onyekwelu I, et al. Outcomes and revision rates in normal, overweight, and obese patients 5 years after lumbar fusion[J]. Spine Journal, 2016, 16(10):1178-1183.

6. Ahn J, Tabaraee E, Bohl D D, et al. Primary Versus Revision Single-level Minimally Invasive Lumbar Discectomy: Analysis of Clinical Outcomes and Narcotic Utilization[J]. Spine, 2015, 40(18):1025-1030.

7. Park P, Garton H J, Gala V C, et al. Adjacent Segment Disease after Lumbar or Lumbosacral Fusion: Review of the Literature[J]. Spine, 2004, 29(17):1938-1944.

8. Cheh G, Bridwell K, Lenke L, et al. Adjacent segment disease followinglumbar/thoracolumbar fusion with pedicle screw instrumentation: a minimum 5-year follow-up. [J]. Spine, 2007, 32(20):2253-2257.

9. Rodriguez A, Neal M T, Liu A, et al. Novel placement of cortical bone trajectory screws in previously instrumented pedicles for adjacent-segment lumbar disease using CT image-guided navigation[J]. Neurosurgical Focus, 2014, 36(3): E9.

10. Wang YY, Zhang Jf, Fan SW, et al. Application of cortical bone trajectory screw in the revision of lumbar disease[J]. Chin J Orthop, 2017, 37(18): 1143-1149.

11. Chen $\mathrm{C} \mathrm{H}$, Huang $\mathrm{H} \mathrm{M}$, Chen D C, et al. Cortical bone trajectory screws fixation in lumbar adjacent segment disease: A technique note with case series[J]. Journal of Clinical Neuroscience, 2017,48:224-228.

12. Mullin J P, Perlmutter B, Schmidt E, et al. Radiographic feasibility study of cortical bone trajectory and traditional pedicle screw dual trajectories[J]. Journal of Neurosurgery: Spine, 2016, 25(6):727-732.

13. Ueno M, Imura T, Inoue G, et al. Posterior corrective fusion using a double-trajectory technique (cortical bone trajectory combined with traditional trajectory) for degenerative lumbar scoliosis with osteoporosis[J]. Journal of Neurosurgery: Spine, 2013, 19(5):600-607. 


\section{Figures}
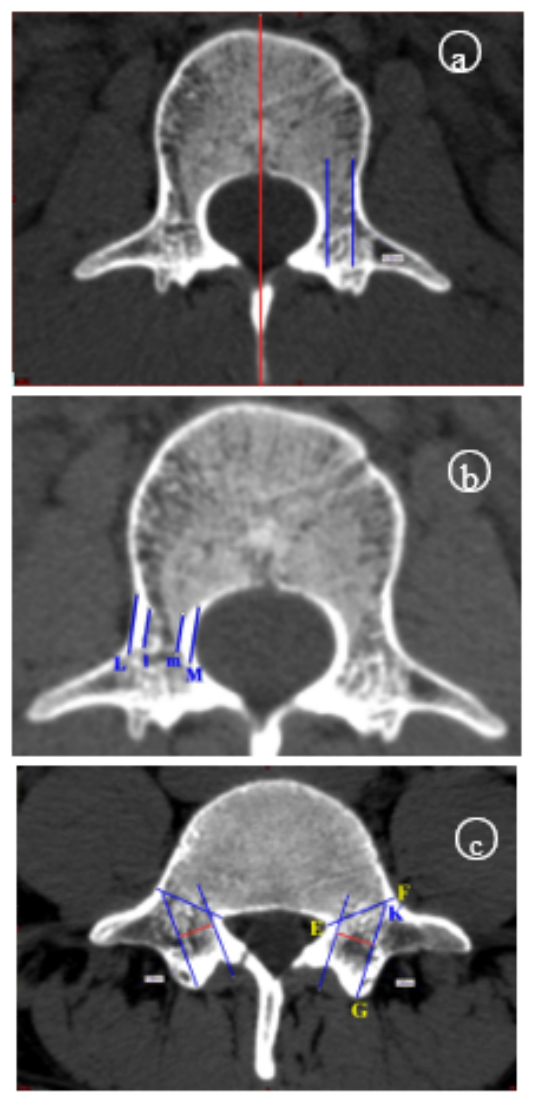

\section{Figure 1}

a. CBT width in L1-L4 pedicles. A plain scan with the widest lateral wall of the pedicle is selected. Straight lines are created parallel to the mid-line of the vertebral body at the medial edge of the lateral wall and the lateral edge of the medial wall of the pedicle to measure the distance between the two lines. b. Schematic of the TRW of the L5 pedicle. A line is drawn at the narrowest width of this plane (line EF). This line intersects the medial edge of the lateral wall at point $\mathrm{K}$. Then, the lateral ridge of the lamina is identified and marked as point $\mathrm{G}$. A line parallel to the line $\mathrm{GK}$ is drawn at the lateral edge of the medial wall. Finally, the distance between the two parallel lines is measured. c. A plain scan with the widest lateral wall of the pedicle is first selected, and then the narrowest width in the crosssection is selected. Two parallel lines are drawn along the pedicle axis ( $L$ and $M)$ at lateral edge of lateral wall and at medial edge of medial wall of the pedicle. The distance between lines $L$ and $M$ is the PW. Two parallel lines are drawn along the pedicle axis ( $\mathrm{l}$ and $\mathrm{m}$ ) at medial edge of lateral wall and at lateral edge of medial wall of the pedicle. The distance between lines I and $\mathrm{m}$ is the SBW. 

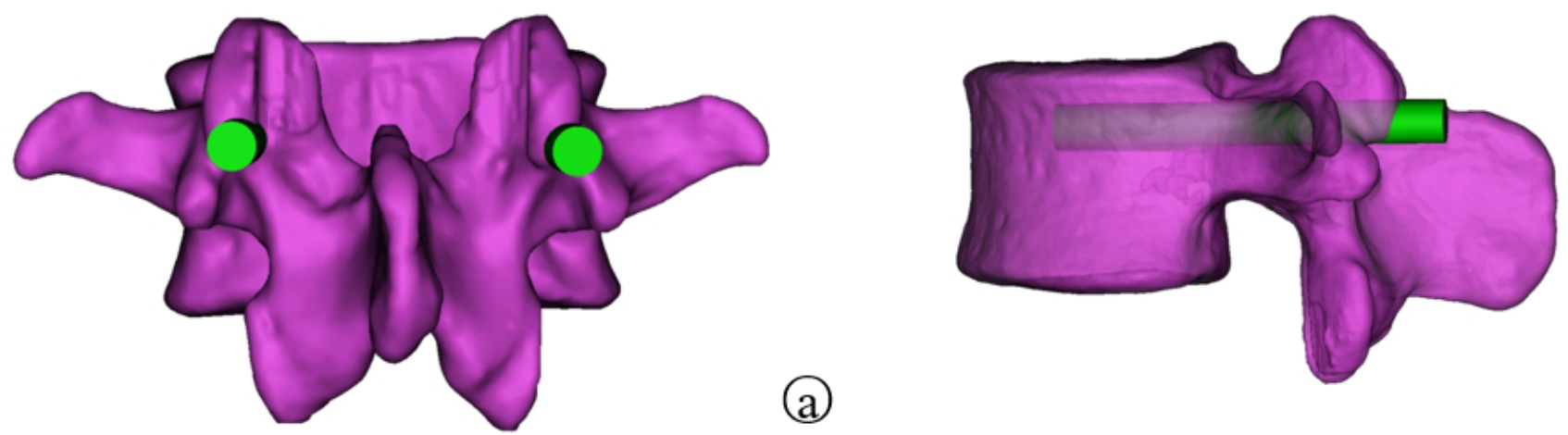

(a)

(b)

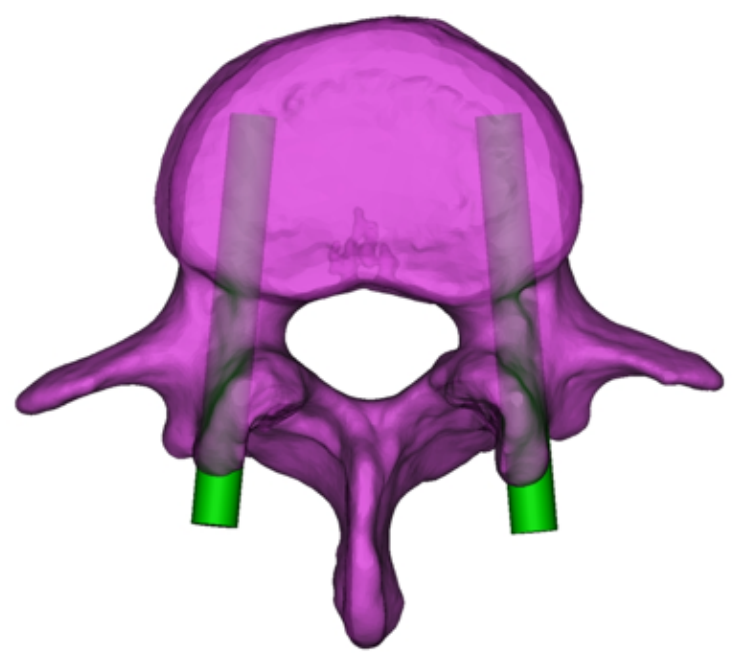

(c)

\section{Figure 2}

The criteria of the TP screw placement. a. The screw entry point is located at the vertex point of the " $\lambda$ "-shaped ridge. b. The screw trajectory is close to the upper edge of the pedicle and parallel to the endplate of the vertebral body. c. The screw is placed along the pedicle axis in the cross section, and the depth is $1 / 3$ of the vertebral body. 

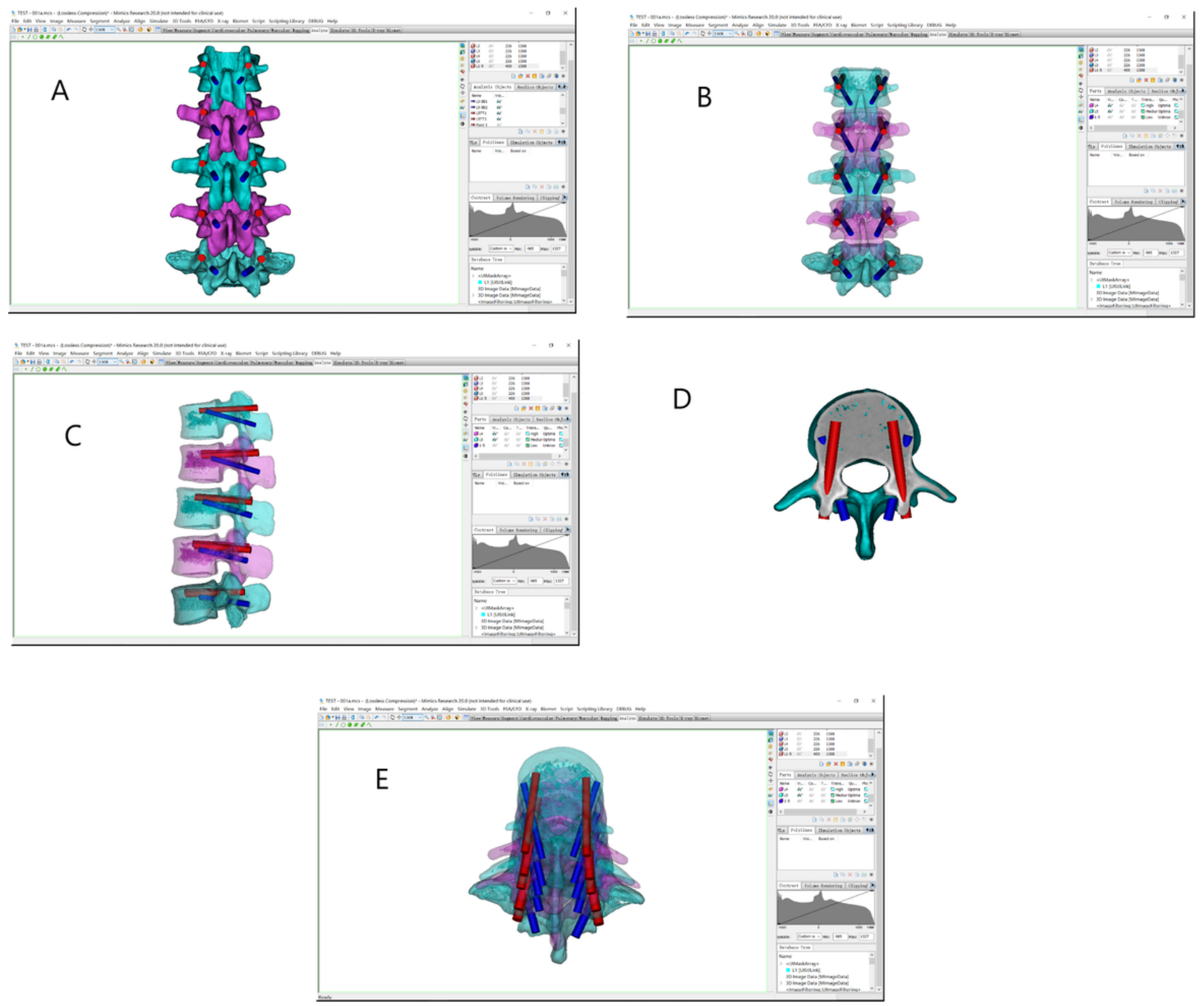

\section{Figure 3}

Schematic of the CBT screw placement. a. The TP screw is placed at the vertex of the " $\lambda$ "-shaped ridge. The CBT screw placement site is located at the farther medial-inferior site. $b$. The anteroposterior view of the vertebral body (transparent mode) shows the positional relationship of the 2 screws. c. The lateral view shows the positional relationship of the 2 screws. $d$. The axial view shows that the CBT screw is located under the pedicle screw, and the tip of the CBT screw reaches the sidewall of the vertebral body. e. The top view shows the positional relationship of the 2 screws. 


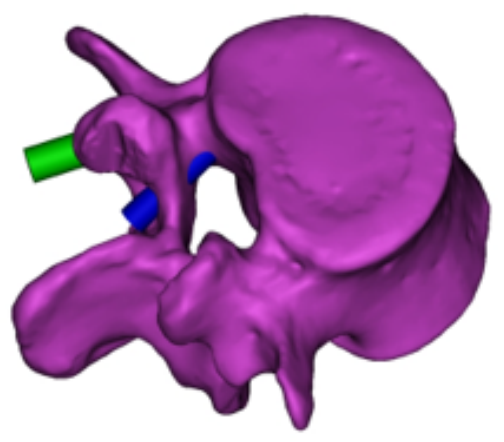

(a)

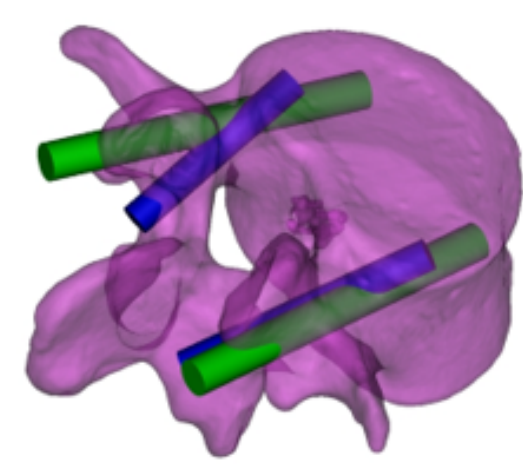

(b)

Figure 4

CBT screw placement with the same TP screw parameters. a. Schematic of CBT screw penetration of the medial wall of the pedicle. b. The view presented in transparent mode shows the overlap of the 2 screws. The images depicted above represent a failed screw placement.

\section{Supplementary Files}

This is a list of supplementary files associated with this preprint. Click to download.

- Declarations.docx 